\title{
A prospective study on feto-maternal outcome in patients with premature rupture of membranes at tertiary care center
}

\author{
Patil S. A. ${ }^{1}$, Gaikwad B. H. ${ }^{2}$, Purohit P. ${ }^{1}$, Chintan Upadhyay ${ }^{1 *}$
}

\begin{abstract}
${ }^{1}$ Department of Obstetrics and Gynecology, ND Desai Medical College, Nadiad, Gujarat, India
${ }^{2}$ Department of Obstetrics and Gynecology, RCSM GMC, Kolhapur, Maharashtra, India
\end{abstract}

Received: 31 December 2020

Accepted: 04 February 2021

\author{
*Correspondence: \\ Dr. Chintan Upadhyay, \\ E-mail: drchintan1508@gmail.com
}

Copyright: (C) the author(s), publisher and licensee Medip Academy. This is an open-access article distributed under the terms of the Creative Commons Attribution Non-Commercial License, which permits unrestricted non-commercial use, distribution, and reproduction in any medium, provided the original work is properly cited.

\begin{abstract}
Background: PROM is associated with increased risk of chorioamnionitis, unfavorable cervix and dysfunctional labour, increased cesarean rates, postpartum hemorrhage and endometritis in the mother. Possible neonatal outcomes in cases of PROM may include respiratory distress syndrome, hypothermia, hypoglycemia, intraventricular hemorrhage, broncho pulmonary dysplasia etc. Objective of this study the maternal and perinatal outcomes in premature rupture of membranes at term.

Methods: This prospective study was done among 100 pregnant women with premature rupture of membranes after 37 completed weeks visited at department of obstetrics \& Gynecology in RCSM Hospital, Kolhapur during June 2014 and June 2015. Inclusion Criteria was Gestational age of $>37$ weeks confirmed by dates, clinical examination or ultrasound, cervical dilatation of $<3 \mathrm{cms}$, Lack of uterine contractions for atleast 1 hour from PROM, Single live pregnancy in vertex presentation and PROM confirmed by Direct visualization or Litmus.

Results: Present study found highest number of cases among age group 20-24 years and mean age was 22 years. Almost $70 \%$ cases were un-booked. Average duration to PROM to hospital admission was 9.6 hours. Most common maternal outcome was febrile illness Most common perinatal outcome was birth asphyxia and 3 perinatal death.

Conclusions: Pregnancies complicated with PROM should have supervised labor preferably in an institution. Management of each case has to be individualised. A combined effort of obstetrician and neonatalogist is necessary. A good neonatal intensive care unit can be instrumental in reducing the perinatal morbidity and mortality.
\end{abstract}

Keywords: Fetomaternal Outcome, LSCS, Multigravida, PROM, Primigravida

\section{INTRODUCTION}

The normal development, structural integrity and function of the fetal membranes are essential for the normal progress and outcome of pregnancy. One of the most important functions of the membranes is to remain intact till the labour starts in order to maintain the protective intrauterine fluid environment. ${ }^{1}$

Premature rupture of membranes (PROM) refers to a patient who is beyond 37 weeks' gestation and has presented with rupture of membranes (ROM) prior to the onset of labor. Preterm premature rupture of membranes
(PPROM) is ROM prior to 37 weeks' gestation. Spontaneous premature rupture of the membranes (SPROM) is ROM after or with the onset of labor. Prolonged ROM is any ROM that persists for more than 24 hours and prior to the onset of labor PROM occurs in approximately $10 \%$ of pregnancy. ${ }^{2,3}$

PROM is characterized by rupture of membranes before the onset of true labour. This occurs in 5-20\% of all labours. Indian studies report an incidence of PROM in 7$12 \%$ of all labours. $70 \%$ of the cases it occurs in pregnancies at term. ${ }^{4} \mathrm{PROM}$ is associated with increased risk of chorioamnionitis, unfavorable cervix and 
dysfunctional labour, increased cesarean rates, postpartum hemorrhage and endometritis in the mother. ${ }^{5,6}$

Possible neonatal outcomes in cases of PROM may include respiratory distress syndrome, hypothermia, hypoglycemia, intraventricular hemorrhage, broncho pulmonary dysplasia etc. PROM is associated with $20 \%$ of neonatal deaths. Hence PROM is an obstetric condition which is poorly defined with an obscure etiology and associated with significant maternal morbidity and mortality ${ }^{7}$. So, the present study was conducted with the objective to study the maternal and perinatal outcomes in premature rupture of membranes at term.

\section{METHODS}

This prospective study was done among 100 pregnant women with premature rupture of membranes after 37 completed weeks visited at department of obstetrics \& Gynecology in RCSM Hospital, Kolhapur during June 2014 and June 2015. Data collection was done after ethical permission from institutional ethical committee and informed consent of clients. Inclusion Criteria was Gestational age of >37 weeks confirmed by dates, clinical examination or ultrasound, Cervical dilatation of $<3 \mathrm{cms}$, Lack of uterine contractions for atleast 1 hour from PROM, Single live pregnancy in vertex presentation and PROM confirmed by Direct visualization or Litmus. Exclusion Criteria was Gestational age <37 weeks, Cervical dilatation of $>3 \mathrm{cms}$, Women in labour or with uterine contractions within 1 hour of rupture of membrane, Previous cesarean section, Malpresentations and multiple gestations and Complications like contracted pelvis, cephalopelvic disproportion, multiple pregnancy and malpresentations.

A detailed menstrual and obstetric history of the case was noted in a separate questionnaire sheet by interviewing. Detailed clinical obstetric examination was done and history of the signs and symptoms were noted which include time of onset of draining, amount of fluid lost, its color, odor, association with pain or bleeding per vagina and perception of fetal movements. General examination and systemic examination was done as per the protocol. The data were recorded in an Excel sheet and descriptive analysis was performed, of which data are presented in the tables. To know the association between dependent and independent variables chi-square was applied accordingly. $\mathrm{P}$ value less than 0.05 was considered as statistically significant.

\section{RESULTS}

Table 1 shows that $14,61,20,3$ and 2 participants belonged to age group 15-19, 20-24, 25-29, 30-34 and $>35$ years respectively. Mean age was 22 years \pm 7.6 SD. Ante-natal registration was done in 31 cases. Study included 63 primigravida and 37 multigravida cases. Time between PROM to admission was 10-6, 6-12, 12-
24, 24-48 and 48 hours in 46, 30, 20, 3 and 1 cases respectively. Mode of delivery was Vaginal delivery, Ventouse delivery and Cesarean section done in 66, 4, 30 cases respectively. Indication for LSCS was Failure to progress, Fetal distress, DTA and Intrapartum sepsis in $15,11,2$ and 2 respectively.

Table 1: Clinico-social factors of study participants $(\mathrm{N}=100)$.

\begin{tabular}{|ll|}
\hline Variable & Number \\
\hline Age (in year) & \\
\hline $15-19$ & 14 \\
\hline $20-24$ & 61 \\
\hline $25-29$ & 20 \\
\hline $30-34$ & 3 \\
\hline$>35$ & 2 \\
\hline Mean age \pm SD & $22 \pm 7.6$ \\
\hline Ante-natal registration & \\
\hline Booked & 31 \\
\hline Un-booked & 69 \\
\hline Gravida & \\
\hline Primi & 63 \\
\hline Multi & 37 \\
\hline Time between PROM to admission (in hour) \\
\hline $0-6$ & 46 \\
\hline $6-12$ & 30 \\
\hline $12-24$ & 20 \\
\hline $24-48$ & 3 \\
\hline 48 & 1 \\
\hline Mean duration \pm SD & $9.6 \pm 3.9$ \\
\hline Mode of delivery & 66 \\
\hline Vaginal delivery & 4 \\
\hline Ventouse delivery & 30 \\
\hline Cesarean section & 15 \\
\hline Indication for LSCS & 2 \\
\hline Failure to progress & \\
\hline Fetal distress & \\
\hline DTA & \\
\hline Intrapartum sepsis & \\
\hline & \\
\hline
\end{tabular}

Table 2 shows that $9.5 \%, 66.7 \%$ and $23.8 \%$ primigravida cases and $0 \%, 40.5 \%$ and $59.5 \%$ multigravida cases have bishop's score 0-2, 3-4 and 5-6 respectively. Association between gravida and bishop's score was statistically significant $[p<0.05]$. Almost 3.1\%, 20.6\%, 68.4\% and $7.9 \%$ primigravida cases and $2.7 \%, 43.2 \%, 48.6 \%$ and $5.4 \%$ multigravida cases have time for Induction to Delivery Interval was 0-6, 6-12, 12-24 and 24-48 hours respectively. Association between gravida and time for Induction to Delivery Interval was statistically not significant [p>0. 05]. Almost 0.0\%, 1.5\%, 73.5\%, 23.5\% and $1.5 \%$ primigravida cases and $2.7 \%, 24.3 \%, 51.4 \%$, $21.6 \%$ and $0.0 \%$ multigravida cases have time for PROM to Delivery Interval was 0-6, 6-12, 12-24, 24-48 and >48 hours respectively. Association between gravida and time for PROM to delivery Interval was statistically significant $[\mathrm{p}<0$. 05]. Almost $63.5 \%, 8.3 \%$ and $31.7 \%$ 
primigravida cases and $70.3 \%, 2.7 \%$ and $27.0 \%$ multigravida cases were delivered baby by vaginal, ventouse and LSCS mode respectively. Association between gravida and mode of delivery was statistically not significant [ $\mathrm{p}>0.05]$.

Table 2: Association between different clinical variables with gravida $(\mathrm{N}=100)$.

\begin{tabular}{|c|c|c|c|}
\hline \multirow{2}{*}{ Variable } & \multicolumn{2}{|l|}{ Gravida } & \multirow{2}{*}{ P value } \\
\hline & Primi $(n=63)(\%)$ & Gravida $(n=37)(\%)$ & \\
\hline \multicolumn{4}{|c|}{ Bishop's score } \\
\hline $0-2$ & $6(9.5)$ & $0(0)$ & \multirow{3}{*}{$<0.05$} \\
\hline $3-4$ & $42(66.7)$ & $15(40.5)$ & \\
\hline $5-6$ & $15(23.8)$ & $22(59.5)$ & \\
\hline \multicolumn{4}{|c|}{ Time for induction to delivery interval (in hours) } \\
\hline $0-6$ & $2(3.1)$ & $1(2.7)$ & \multirow{4}{*}{$>0.05$} \\
\hline $6-12$ & $13(20.6)$ & $16(43.2)$ & \\
\hline $12-24$ & $43(68.4)$ & $18(48.6)$ & \\
\hline $24-48$ & $5(7.9)$ & $2(5.4)$ & \\
\hline \multicolumn{4}{|c|}{ Time for PROM to delivery interval (in hour) } \\
\hline $0-6$ & $0(0)$ & $1(2.7)$ & \multirow{5}{*}{$<0.05$} \\
\hline $6-12$ & $1(1.5)$ & $9(24.3)$ & \\
\hline $12-24$ & $47(73.5)$ & $19(51.4)$ & \\
\hline $24-48$ & $15(23.5)$ & $8(21.6)$ & \\
\hline$>48$ & $1(1.5)$ & $0(0)$ & \\
\hline \multicolumn{4}{|c|}{ Mode of delivery } \\
\hline Vaginal & $40(63.5)$ & $26(70.3)$ & \multirow{3}{*}{$>0.05$} \\
\hline Ventouse & $3(8.3)$ & $1(2.7)$ & \\
\hline LSCS & $20(31.7)$ & $10(27)$ & \\
\hline
\end{tabular}

* - Chi-square Test

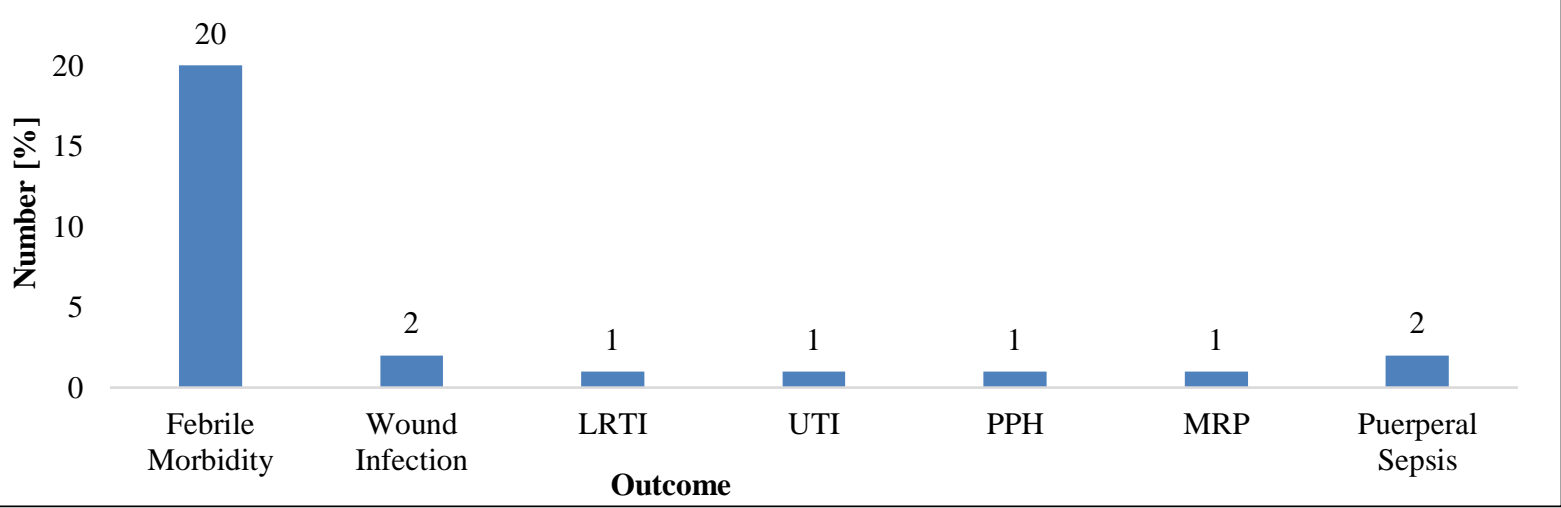

Figure 1: Maternal outcome.

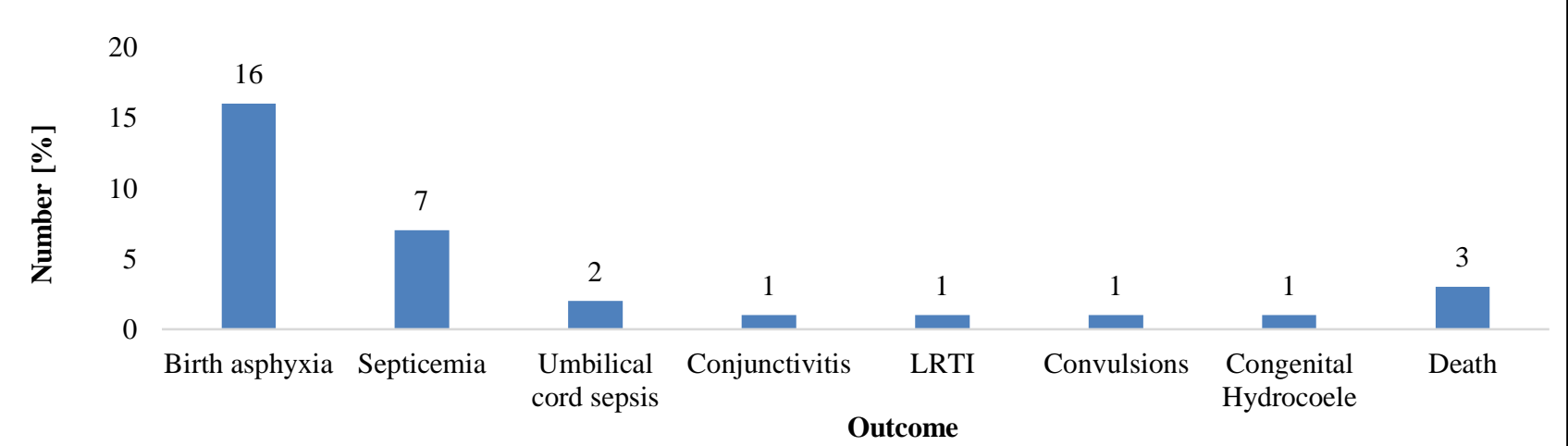

Figure 2: Perinatal outcome. 
Figure 1 shows that maternal outcome like febrile morbidity, wound infection, LRTI, UTI, PPH, MRP and puerperal sepsis was observed in 20, 2, 1,1,1,1 and 2 cases respectively. Figure 2 shows that birth asphyxia, septicaemia, umbilical cord sepsis, conjunctivitis, LRTI, convulsion, congenital hydrocele and death observed in $16,7,2,1,1,1,1$ and 3 cases respectively.

\section{DISCUSSION}

PROM is an enigmatic condition associated with high risk of maternal morbidity, perinatal morbidity and mortality. It complicates $5-10 \%$ of all pregnancies. Complication increase with decrease in gestational age and increase in the latent period. ${ }^{4}$

Present study found highest number of cases among age group 20-24 years and mean age was 22 years. This finding is comparative with the study done by Gaikwad BH et al, Nagaria $\mathrm{T}$ et al. ${ }^{4,8}$ Our study observed that almost $70 \%$ cases were un-booked which correlate with the study done by Nagaria $\mathrm{T}$ et al and Shah $\mathrm{M}$ et al. ${ }^{8,9}$ According to Revathi et al and Malay Sarkar et al, the poor antenatal booking has got a significant role in the risk factors on PROM. ${ }^{1}$ Our study observed that average duration to PROM to hospital admission was 9.6 hours. Study done by Gaikwad BH et al observed same duration was 12.1 hours and Surayapalem $S$ et al. ${ }^{4,10}$ Almost $2 / 3^{\text {rd }}$ cases were delivered baby by vaginal route and around $1 / 3^{\text {rd }}$ cases were by LSCS. Similar observation was also observed by Devi A et al, Singhal P et al, Kamala J et al and Ray $\mathrm{P}$ et al. ${ }^{11-14}$

Most patients (90\%) enter spontaneous labour within 24 hours when they experience ROM at term. Eighty-five percent of neonatal morbidity and mortality is a result of prematurity. PPROM is associated with $30-40 \%$ of preterm deliveries and is the leading identifiable cause of preterm delivery. The major question regarding management of these patients is whether to allow them to enter labor spontaneously or to induce labor. ${ }^{8}$

Present study observed significantly higher bishop's score in multi-gravida cases. Our study also observed that significantly higher duration between PROM to delivery among primi cases compare to multi gravida cases. Our study not observed any maternal death during study period. Most common maternal outcome was febrile illness. Study done by Amla $\mathrm{S}$ et al observed most common maternal outcome was post-partum fever and UTI. $^{1}$

Present study observed 3 perinatal death during study period, in which two death due to birth asphyxia and one death due to septicemia. Most common perinatal outcome was birth asphyxia. Similar finding was observed by Gaikwad BH et al, Devi A et al and Singhal P et al. ${ }^{4,11,12}$ Amala $\mathrm{S}$ et al observed most common outcome was Low birth weight [LBW] and respiratory distress. ${ }^{1}$

\section{CONCLUSION}

PROM is an enigmatic condition associated with high risk of maternal morbidity, perinatal morbidity and mortality. It complicates $5-10 \%$ of all pregnancies. Complications increase with decrease in gestational age and increase in the latent period. Difficulties are found in the diagnosis of PROM. Recognition of etiological factors and management. Pregnancies complicated with PROM should have supervised labor preferably in an institution. Management of each case has to be individualised. A combined effort of obstetrician and neonatalogist is necessary. A good neonatal intensive care unit can be instrumental in reducing the perinatal morbidity and mortality. Thus a team approach, early recognition of premature rupture of membranes and their associated complication and appropriate. Management of situation helps in reducing the problems caused by PROM to a great extent.

Funding: No funding sources

Conflict of interest: None declared

Ethical approval: The study was approved by the Institutional Ethics Committee

\section{REFERENCES}

1. Amala S, Kumari KL. A Study of Maternal and Perinatal Outcome in Pre Labour Rupture of Membranes at Term. JMSCR. 2017;5(11):3022530230.

2. Frenette P, Dodds L, Armson BA, Jangaard K. Pretermprelabour rupture of membranes: effect of latency on neonatal and maternal outcomes J Obstet Gynaecol Can. 2013;35(8):710-7.

3. Acaia B, Crovetto F, Ossola MW, Nozza S, Baffero GM, Somigliana E, et al. Predictive factors for neonatal survival in women with periviable preterm rupture of the membranes. J Matern Fetal Neonatal Med. 2013;26(16):1628-34.

4. Gaikwad BH, Maskar RP. Study of maternal and perinatal outcome in premature rupture of membrane at term pregnancy. J Evolution Med Dent Sci. 2016;5(37):2226-8.

5. Raghavan SS. Endocervical prostaglandin E2 (PGE2) gel in premature rupture of membranes. J Obstet Gynecol India. 2001;51:122.

6. Uma S, Nisha $S$, Shikha S. A prospective analysis of etiology and outcome of preterm labor. J Obstet Gynecol India. 2007;57(1):48-52.

7. Naeye R, Peters E. Causes and consequences of premature rupture of fetal membranes. Lancet. 1980;1:192-4.

8. Nagaria T, Diwan C, Jaiswal J. A study on fetomaternal outcome in patients with premature rupture of membranes. Int $\mathbf{J}$ Reprod Contracept Obstet Gynecol. 2016;5:4123-7.

9. Shah M, Sandesara P. Fetomaternal outcome in cases of PROM. Gujarat Med J. 2011;66(1). 
10. Surayapalem S, Cooly V, Salicheemala B. A study on maternal and perinatal outcome in premature rupture of membranes at term. Int $\mathbf{J}$ Reprod Contracept Obstet Gynecol. 2017;6:5368-72.

11. Devi A, Reddi Rani. Premature rupture of membranes - A clinical study. J Obstet Gynaecol India. 1996;46:63.

12. Singhal P, Singhal AK. Fetomaternal outcome in premature rupture of membranes. Obs Gyna Today. 2002;10:585.
13. Kamala J, Scaila S. A study of premature rupture of membranes Management and outcome. J Obstet Gynaecol India. 2001;51(2):58-60.

14. Ray P, Sikdar K, Das A, Ghosh T. Study of cases of chorioamniotics followed premature rupture of membranes. J Obstet Gynaecol India. 1997;47:136.

Cite this article as: Patil SA, Gaikwad BH, Purohit $\mathrm{P}$, Upadhyay C. A prospective study on feto-maternal outcome in patients with premature rupture of membranes at tertiary care center. Int J Reprod Contracept Obstet Gynecol 2021;10:1022-6. 